\title{
Snow and ice applications of AVHRR in polar regions: report of a workshop held in Boulder, Colorado, 20 May 1992
}

K. STEFFEN
Cooperative Institute for Research in Environmental Sciences, University of Colorado, Campus Box 449, Boulder, CO 80309, U.S.A.

R. BINDSCHADLER

NASA/Goddard Space Flight Center, Code 971, Greenbelt, MD 20771, U.S.A.

G. Casassa

Byrd Polar Research Center, Ohio State University, 125 South Oval Mall, Columbus, OH 43210-1308, U.S.A.

J. Comiso

NASA/Goddard Space Flight Center, Code 971, Greenbelt, MD 20771, U.S.A.

D. EPPLER

Polar Oceanography Branch, NOARL/CRREL, 72 Lyme Road, Hanover, NH 03755-1294, U.S.A.

F. FETTERER

Remote Sensing Branch, NOARL-Stennis Space Center, Bay St. Louis, MS 39529-5004, U.S.A.

J. HAWKINS

Naval Research Laboratory, Monterey, CA 93943, U.S.A.

J. KEY

Cooperative Institute for Research in Environmental Sciences, University of Colorado, Campus Box 449, Boulder, CO 80309, U.S.A.

\section{ROTHROCK}

University of Washington, Applied Physics Lab, Polar Science Center, 1013 NE 40th Street, Seattle, WA 98105, U.S.A.

\section{R. THOMAS}

NASA Headquarters, Code SED, Washington, DC 20546, U.S.A.

\section{R. WEAVER}

Cooperative Institute for Research in Environmental Sciences, University of Colorado, Campus Box 449, Boulder, CO 80309, U.S.A.

\section{R. WELCH}

South Dakota School of Mines and Technology, 501 E St. Joseph Street, Rapid City, SD 57701-3995, U.S.A.

\begin{abstract}
The third symposium on Remote Sensing of Snow and Ice, organized by the International Glaciological Society, took place in Boulder, Colorado, 17-22 May 1992. As part of this meeting a total of 21 papers was presented on snow and ice applications of Advanced Very High Resolution Radiometer (AVHRR) satellite data in polar regions. Also during this meeting a NASA sponsored Workshop was held to review the status of polar surface measurements from AVHRR. In the following we have summarized the ideas and recommendations from the workshop, and the conclusions of relevant papers given during the regular symposium sessions. The seven topics discussed include cloud masking, ice surface temperature, narrow-band albedo, ice concentration, lead statistics, sea-ice motion and ice-sheet studies with specifics on applications, algorithms and accuracy, following recommendations for future improvements. In general, we can affirm the strong potential of AVHRR for studying sea ice and snow covered surfaces, and we highly recommend this satellite data set for long-term monitoring of polar process studies. However, progress is needed to reduce the uncertainty of the retrieved parameters for all of the above mentioned topics to make this data set useful for direct climate applications such as heat balance studies and others. Further, the acquisition and processing of polar AVHRR data must become better coordinated between receiving stations, data centers and funding agencies to guarantee a long-term commitment to the collection and distribution of high quality data.
\end{abstract}




\section{INTRODUCTION}

The Advanced Very-High Resolution Radiometer (AVHRR) is a satellite sensor with strong potential for studying sea ice and snow covered ice surfaces. Its visible and infrared channels, listed in Table 1, offer data with direct applicability to problems of heat balance. Its spatial resolution of $1 \mathrm{~km}$ at nadir elucidates the complex structures of leads and floes of sea ice and flowbands of ice sheets. Its frequent polar coverage and broad swath are sufficient to resolve synoptic and even diurnal changes of surface temperature and lead patterns, and to track seaice motion. The decades-long availability and planned continuation of this sensor make it a primary source of data that can be use to monitor global climate.

Table 1. AVHRR wavelengths in microns. Swath width is $2580 \mathrm{~km}$ High Resolution Picture Transmission (HRPT). Ground resolution is $1.1 \mathrm{~km}$ at nadir for Local Area Coverage (LAC) and HRPT. Global Area Coverage $(G A C)$ data have a ground resolution of $4 \mathrm{~km}$ (after Kidwell, 1991)

\begin{tabular}{|c|c|c|c|}
\hline \multirow{2}{*}{\multicolumn{2}{|c|}{ Band Spectral region }} & \multirow{2}{*}{$\begin{array}{c}\text { Wavelength } \\
\text { NOAA- } 6,8,10 \\
\mu \mathrm{m}\end{array}$} & \multirow{2}{*}{$\begin{array}{c}\text { Wavelength } \\
\text { NOAA-7, 9, 11, } 1 \\
\mu \mathrm{m}\end{array}$} \\
\hline & & & \\
\hline 1 & Visible & $0.58-0.68$ & $0.58-0.68$ \\
\hline 2 & Near infrared & $0.725-1.10$ & $0.725-1.10$ \\
\hline 3 & Infrared & $3.55-3.93$ & $3.55-3.93$ \\
\hline 4 & Thermal infrare & ed $\quad 10.50-11.50$ & $10.30-11.30$ \\
\hline 5 & Thermal infrare & ed $10.50-11.50$ & $11.50-12.50$ \\
\hline
\end{tabular}

Despite this promise, AVHRR has not been broadly applied to polar scientific problems. A number of studies have been undertaken to show how geophysically important surface parameters can be inferred using AVHRR data, beginning two decades ago with threeday minimum-brightness estimates of ice concentrations. Most studies, however, have worked in a demonstration and/or case study mode, and have not been developed into a monitoring capability. In contrast, consider the widely accepted data set to study the cloud cover (International Satellite Cloud Climatology Project, ISCCP), the variability of biomass through a vegetation index, and the sea surface temperature. There is no analogy to these data sets for any polar surface variable derived from AVHRR data. A reason for this lack of applications to the polar regions has been and continues to be cloudiness that obscures the surface for most of the summer and much of the winter. Although there are holes in the cloud cover through which the surface can be viewed, differentiating cloud from surface over snow and ice is far more difficult than at lower latitudes where the albedo and temperature of land and water are quite different from those of clouds. Another problem is the diverse number of institutions and agencies where AVHRR data are archived, in multiple formats and media, and for various periods, all without inter-related catalogs. For this reason, the utilization of polar AVHRR data is poor.

There is some feeling that the polar community needs better access to polar-gridded, calibrated, cloud-masked, and geolocated Local Area Coverage (LAC)/High Resolution Picture Transmission (HRPT) data. The Office of Naval Research has supported the development of such a data set for most of the Arctic Ocean covering the year 1989, selected for best compromise between coverage and cloudlessness. Subsequent data are now being processed, and will be available through the National Snow and Ice Data Center in Boulder, Colorado. Prototyping of data for NASA's planned MODIS sensor requires a high resolution AVHRR data set; what polar data should be included? The re-analysis and archiving of older global data sets are being supported by NASA under the Pathfinder program; no high resolution polar AVHRR data are presently included. Before arguments can be made for better archived polar data sets, the utility of such data must be assessed, and a justification made. There is something of a chicken-andegg problem here: better data sets must be justified by both the scientific needs and maturity of algorithms, yet the demonstration of the data's utility requires improved data access.

To begin to resolve this stalemate, we have initiated a review of the status of polar surface measurements from AVHRR. As part of this process we have posed the following questions, solid answers to which will define limitations, strengths, and uses for these data.

1. What are the most important polar surface parameters that can be derived from the AVHRR data, and by what algorithms are they estimated?

2. How accurately can each be derived? What steps could be taken to improve the accuracy?

3. What validation is needed before these parameters can be estimated accurately and routinely for scientific research?

4. What are appropriate sampling objectives? What are the optimal spatial resolutions for each of the parameters? For what applications are lower resolution Global Area Coverage (GAC) data useful? For which are higher resolution LAC or HRPT necessary? What temporal resolution and regional coverage are required?

5. What difficulties are encountered by users, and how can they be mitigated? Are calibration and geolocation data adequate? How can the barrier of cloudiness be dealt with? Are needed data available through current archives?

A consideration of the first three of these questions seemed compatible with this International Glaciological Society symposium, so a half-day workshop was scheduled during the meeting. The remaining questions seemed more appropriate for a different group of people than would be gathered at Boulder. Because of limited time, the agenda was focused to the most relevant materials, but even then time for discussion was not adequate. For that, we apologize to the workshop participants, who sacrificed a mountain tour and hike to attend. The workshop format consisted of a review of each of seven 
topics: cloud masking (excluding determination of cloud properties), surface temperature, albedo, ice concentration, lead statistics, ice motion, and ice sheet properties. These seven reviews are presented in the next seven sections of this paper. Rapporteurs (the authors of this report) reported the conclusions of relevant papers given during the regular symposium sessions.

This report summarizes ideas from the general workshop and symposium discussions. To keep the paper to a modest length, we have not expanded it into a full state-of-the-art review of these seven topics, but such a thorough review would be timely. In the final section, we draw attention to the most pressing needs for and most promising developments of quantitative polar applications of AVHRR data.

\section{CLOUD MASKING}

\subsection{Introduction}

Cloud masking is of critical importance in polar regions. Clouds and snow/ice surface features have similar characteristics in both the visible and infrared spectral ranges, thereby making polar scene identification extremely difficult. Often both high level cirrus clouds and low level fog and stratus clouds are optically thin. In such cases, retrievals of surface parameters such as albedo and temperature are seriously compromised.

\subsection{Algorithms}

As part of the International Satellite Cloud Climatology Project (ISCCP), global cloud cover is being retrieved. Typical cloud masking algorithms assume that clouds can be detected using visible and infrared channel thresholds. Reflectance thresholds typically are set about 3\% above the background, and thermal thresholds typically are set about $3^{\circ} \mathrm{C}$ below the background. Other approaches rely upon bispectral thresholding (Minnis and Harrison, 1984) and a variety of statistical methods (e.g. Saunders and Kriebel, 1988). However, Rossow and others (1989), Stowe and others (1989) and many others have reported difficulties associated with polar cloud cover retrievals. Indeed, Landsat imagery shows that clouds often are darker than the background snow and ice (Welch and others, 1992a). In particular, cloud cover often is confused with melt ponds, thin ice and pancake ice. In the infrared spectrum, low surface temperatures, strong inversions and isothermal structure make cloud discrimination difficult. In recent years, it has been shown that combinations of spectral and textural measures can be effective for polar cloud classification (Ebert, 1987; Key, 1990a; Welch and others, 1992a). Spectral measures typically are based upon AVHRR channels 1, 3 and 4. Textural measures typically are computed from channels 1 and 4 ; the $3.7 \mu \mathrm{m}$ channel (channel 3 ) is often too noisy for reliable textural measures. Difference channels, such as ch1-ch2 and ch3-ch4, and ratio channels, such as ch3/ chl, also can be effective for polar scene identification.

During the IGS symposium, thirteen groups presented a variety of approaches to cloud masking, with various levels of success. These techniques can be loosely categorized as: (1) spectral thresholding; (2) statistical and clustering; (3) textural; and (4) spectral and textural. On one extreme, Lindsay and Rothrock (1993) relied upon subjective cloud masking. Serreze and others (1993) reported problems with the ISCCP cloud detection algorithm, and Emery and Fowler (unpublished) tried a number of cloud masking schemes, but reported that none was definitive. Liger and Fily (1993) used the $11 \mu \mathrm{m}$ thermal and $6.7 \mu \mathrm{m}$ water vapor channels, but found that this combination was not very successful. Maslanik and Key (1993) combined AVHRR and microwave SSM/I data to improve their retrievals.

Eppler and Full (1992) applied a Sobel edge gradient measure based upon $3 \times 3,5 \times 5$ and $7 \times 7$ convolution masks, while Comiso (unpublished) used a statistical filtering approach. Massom and Comiso (in press) used statistical measures based upon ch4 vs ch3-ch4 for cloud/ice discrimination, ch4 vs. ch2-ch4 for ice type discrimination. Aizen and Loktionova (unpublished) used channels 3 and 4 not only to discriminate cloud cover, but also to retrieve cloud optical thickness and aerosol properties.

Gallegos and others (1993) used cluster shade (3rd moment) and data degradation for AVHRR cloud masking, and Rees and Lin (1993) applied textures generated from the co-occurrence matrix and fractal measures to classify Landsat MSS scenes. Welch and others (unpublished) applied more than 200 spectral and textural measures within an artificial intelligence classifier. Accuracies greater than $85 \%$ were reported with this approach. Finally, Chou and others (1993) reported a segmentation approach to discriminate cloud and surface features.

Most of the techniques reported during the IGS symposium performed adequately on limited datasets. However, only one group provided objective analysis of classification accuracy. Clearly, before an operational polar cloud masking scheme can be validated and accepted by the polar research community, it is essential that objective measures of accuracy be provided. Typically, the Monte Carlo (e.g., Ebert, 1987) and Bootstrap (Welch and others, unpublished) approaches are used for objective analysis.

There are three main factors that must be addressed in the development of an operational polar cloud masking algorithm: (1) the choice of the feature vector, (2) the choice of the classifier, and (3) proper identification and labeling of regions. First, from the wide range of techniques employed by different groups, it can be seen that there is no consensus as to what types of signatures are needed in a robust polar cloud/surface feature classification scheme. However, it is generally recognized that spectral information alone is inadequate. Difference and ratio channels and textural measures need to be explored further to determine if there is an optimum feature vector for polar scene identification. In this regard, Gray Level Difference Vector textural measures seem to offer a good combination of discriminating ability with low storage and CPU requirements.

There is no consensus as to the size of the region over which textures should be computed. At present $16 \times 16$ pixel regions seem to be preferred. Selection of regions that are too small leads to unstable textural measures. 
However, selection of regions which are too large leads to loss of information; i.e., the region is unlikely to contain a single class. The $16 \times 16$ pixel region seems to provide the best compromise between textural stability and discrimination of features. As a caveat it should be noted that textural measures based upon third order (cluster shade) and fourth order (cluster prominence) statistics generally do not attain textural stability with $16 \times 16$ pixel templates; considerably larger template sizes are required. Therefore, these measures should be applied only after stability analysis is made.

There may not be an optimum feature vector for polar scene identification. Indeed, there seem to be a number of combinations of features that are effective for cloud masking and classification, a few guidelines are emerging. It should be noted that AVHRR channel 3 is particularly valuable in discriminating between water and ice surface features and between water and ice clouds. Also, reflectance values should be divided by the cosine of the solar zenith angle. This procedure normalizes reflectances from different parts of one scene and from different scenes. However, this approach may not be adequate for an operational polar scene classification algorithm. Even normalized ocean and ice reflectance values are observed to vary substantially across a single scan line. This opens the question as to whether bi-directional reflectance functions must be used to normalize the spectral signatures. If so, then a preliminary classification of each region first must be made, followed by the application of the appropriate bi-directional reflectance function. Finally, the solution is iterated to stability. Obviously, for large regions composed of mixed classes, this procedure can be tedious. Furthermore, the bi-directional reflectance functions are known only approximately for most classes.

It should be noted that the cloud classification and cloud masking algorithms presented at this symposium do not cover the entire range of ideas that are found in the literature. For example, it may be possible to utilize TIROS Operational Vertical Sounder (TOVS) or High Resolution Infrared Sounder (HIRS) data effectively, especially for high cloud cover. Another idea which has strong merit is the coupling of AVHRR and passive microwave retrievals. In spite of their large footprints, the microwave channels can provide positive identification of surface features. Another approach which has been suggested is based upon temporal analysis. It relies upon image subtraction to identify regions which have changed from one image to another (i.e., cloud motion). Of course, one must be careful not to confuse sea-ice motion and recent snowfall as cloud cover. Note also that virtually all of the methods presented both at this symposium and in the literature are effective only during the daylight hours. To date an effective "night-time" algorithm has not been demonstrated.

The many groups working on the cloud masking problem have used a spectrum of classification schemes. It is typical to apply some variant of the Maximum Likelihood or Discriminate Analysis approaches. However, it was demonstrated at the symposium that artificial intelligence (AI) classifiers can significantly increase classification accuracy. The main drawbacks of the AI schemes are that these methods are not well understood by most groups and that the training time may be longer than for traditional approaches. On the other hand, in operational mode the AI approaches are very fast. It is also worth noting that the AI approaches are both nonlinear and nonparametric. The advantage is that no particular class structure (i.e., normal distribution) is assumed; rather, the classifier learns through presentation of examples. The AI approaches also require far fewer training samples than do traditional schemes. The new AI approaches presented at this symposium provide very high classification accuracies. The most impressive of these techniques is the "Don't Care" Back Propagation neural network (Welch and others, unpublished). Indeed, for regions containing pure classes, the accuracy exceeds $95 \%$. Nevertheless, much more work is needed to extend these results to mixed classes.

A final issue concerns scene identification and labeling. No classification scheme can be expected to produce accurate results if the labeling is incorrect. The old adage "garbage in, garbage out" is especially appropriate here. The analyst needs to examine a wide variety of information before labeling a region. A new Interactive Visual Image Classification System (IVICS) was introduced at the workshop which provides a wide variety of analysis tools to the user (Welch and others, unpublished). Only using this system have the very high classification accuracies $(>95 \%)$ been attained. The analyst needs to take every precaution in labeling; it is very easy to make labeling errors in polar scene analysis.

\subsection{Recommendations}

In terms of climate change, cloud classification in terms of cloud types, such as stratocumulus, cumulus, and cirrus, is somewhat artificial. Future schemes should focus upon the retrieval of cloud optical depth, phase (water or ice), effective particle size and cloud cover rather than upon cloud type. These are the parameters which directly impact climate change. Similarly, aerosol size distributions and optical depths need to be recovered, at least over water surfaces. Knowledge of the optical properties of aerosol, diamond dust, and thin water and ice cloud, coupled with radiative transfer models, will allow the polar research community to retrieve both surface albedo and temperature, as well as other parameters, with far greater confidence.

It is recommended that priority be given to the development of an operational AVHRR cloud masking/ classification scheme during the next three years. Additional research needs to be made on:

1. Computationally efficient and accurate feature vectors need to be determined for an operational polar cloud masking algorithm.

2. Coupling of AVHRR, TOVS, HIRS and microwave (e.g. SSM/I) data to provide positive identification of surface features.

3. Efficient and accurate classification schemes, including AI approaches, have to be tested for pure classes, and additional work is needed to extend these results to mixed classes.

4. Bi-directional reflectance functions in polar regions (snow and ice surfaces) should be determined and 
applied to normalize the spectral signatures.

5. Improved visualization and database tools will be essential for basin-wide applications.

As they are developed, these schemes must be validated. It is recommended that closer ties be established between researchers working on this problem; as a start, all interested parties should apply their algorithms to a common dataset. Researchers then should communicate their findings every six to 12 months to the science community to intercompare their results. If approached this way, and if validating data from field observational programs is made available, we are confident that a reliable $(>90 \%)$ operational polarcommunity cloud masking scheme can be developed in three years for daytime imagery. It is too early to estimate what accuracy night-time cloud masking approaches may achieve.

\section{ICE SURFACE TEMPERATURE}

\subsection{Application}

One parameter needed for the estimation of radiative and turbulent heat fluxes from satellite data is surface temperature. Little effort has been directed to the retrieval of ice surface temperature (IST) in the Arctic. The reason is not one of methodology, but rather our limited knowledge of atmospheric temperature, humidity, and aerosol vertical, spatial, and temporal distribution, the micro-physical properties of polar clouds, and the spectral characteristics of snow, ice, and water surfaces. Over the open ocean the surface is warm, dark, and relatively homogeneous. This makes sea surface temperature (SST) retrieval, including cloud clearing, a fairly straightforward task. Over the ice, however, the surface within a single AVHRR pixel is likely to be highly heterogeneous, with the spatially integrated emissivity dependent upon the exact mixture of ice of various thicknesses, open water, and snow cover. In addition, Arctic cloud amounts are high (typically from 60-90\%) complicating IST retrieval even further.

\subsection{Algorithms}

The general approach to estimating surface temperatures from thermal channels is to relate satellite observations to surface temperature observations with a regression model. Massom and Comiso (in press) have followed this approach regressing brightness temperatures from the AVHRR against station temperatures in the Bering and Greenland seas. The estimated surface temperatures have an accuracy of $1-3 \mathrm{~K}$.

Lacking a sufficient quantity of corresponding satellite and surface observations, however, satellite radiances or brightness temperatures can be modeled by forward calculation of the radiative transfer equation. This approach is commonly used for SST retrieval (e.g., McClain and others, 1985). SST retrievals with two "split-window" channels have been shown to have an absolute accuracy as high as $0.5-1 \mathrm{~K}$ (RMS error). Land surface temperature (LST) estimates commonly follow the same approach but are generally less accurate due to the larger variability of surface conditions, and errors of 2-3 $\mathrm{K}$ are common. This method of SST retrieval has been employed for IST retrieval from AVHRR data by Key and Haefliger (1992). Temperature and humidity data from Soviet ice islands, skin temperatures estimated with an energy balance model, and modeled directional emissivities of snow were used in the simulation of AVHRR radiances. A regression model was developed that includes the two split-window channels and satellite scan angle. RMS error in the estimated ice surface temperature is stated to be less than $0.1 \mathrm{~K}$ in all seasons. However, this value is based on the regression analysis of the modeled data only; it does not include measurement error, emissivity differences due to mixed pixels, or unmodeled atmospheric effects such as ice crystal precipitation (ICP). In fact, Lindsay and Rothrock (1993), who have applied the same coefficients to Arctic AVHRR data, report the AVHRR ISTs to be biased by about $+4 \mathrm{~K}$ in January 1989 based on comparisons with drifting buoys. They speculate that this large an error can be attributed primarily to the presence of "diamond dust" (ICP).

The basic SST approach described above was also taken by Haefliger and others (1993) with AVHRR data over the Greenland ice sheet. Temperature and humidity profiles from a research site on the Greenland ice sheet were used in the analysis. Skin temperatures were estimated based on upwelling longwave radiation. These same data were used for validation. Surface temperatures were estimated to within $0.5 \mathrm{~K}$ in the worst case, with a mean of about $-0.2 \mathrm{~K}$.

While not utilizing AVHRR data directly, Maslanik and Key (1993) presented preliminary results on the use of SSM/I passive microwave data for IST estimation. The coefficients from Key and Haefliger (1992) were used to estimate the surface temperature within $25 \mathrm{~km}$ cells in winter and then converted to ice temperatures assuming $\mathrm{SSM} / \mathrm{I}$-derived sea-ice concentrations. These ice temperatures were then used to derive an "effective emissivity" for each SSM/I channel, which relates the physical temperature at some frequency-dependent depth within the snow/ice pack to the skin temperature. While errors up to $4 \mathrm{~K}$ were reported, greater accuracy would probably result if the effective emissivities were determined for smaller regions with more homogeneous emissivities and if SSM/I swath data were used rather than the daily averages.

Finally, although not discussed during the conference, the cloud product of the ISCCP contains global surface temperature estimates. For the polar regions these are based on AVHRR data. At the time of this writing, data are available from mid-1983 through 1990. The procedure involves a radiative analysis for each pixel of a reduced-resolution product, and therefore differs from the regression methods described above. Temperatures appear to have a positive bias of a few degrees in winter, although this will probably improve when the original data are reprocessed with a special polar cloud detection algorithm (personal communication from $\mathrm{W}$. Rossow, 1992). 


\subsection{Recommendations}

The difficulties in retrieving IST from AVHRR thermal sensors are not related to methodology per se, but rather to our inadequate knowledge of the atmospheric and surface conditions encountered in the polar regions. Are current estimates accurate enough calculating energy fluxes over ice-covered waters for monitoring climate change? In terms of modeled accuracy only, IST can be retrieved to within $0.1-0.2 \mathrm{~K}$. A more realistic estimate, which includes uncertainties in the measurement and physical (atmospheric and surface) systems, is probably $1-4 \mathrm{~K}$. This translates into an error in upwelling longwave flux of $3-11 \mathrm{~W} \mathrm{~m}^{-2}$ at $230 \mathrm{~K}$ or $5-18 \mathrm{~W} \mathrm{~m}^{-2}$ at $270 \mathrm{~K}$. These values are of the same magnitude as expected changes that we need to detect, so that improvements are certainly required.

In order to obtain more accurate estimates of IST over the polar regions we therefore suggest the following:

1. Cloud masking methods (including detection of ice crystal precipitation) should be improved and automated over snow and ice surfaces, especially during the dark months.

2. Additional information and better understanding of the spatial and temporal distributions of ice crystal precipitation and aerosols are needed. This particular atmospheric phenomenon presents perhaps the greatest uncertainty in IST retrievals from thermal data during late fall, winter, and early spring. It is difficult to detect by its spectral characteristics alone.

3. During summer the water emissivity value should be used mainly to reflect the radiative properties of melting ice, melt ponds and open water. During winter IST retrieval errors due to the emissivity difference of snow and water are less than $0.2 \mathrm{~K}$ and therefore can be neglected at a first approximation.

4. While a considerable amount of surface temperature data has been collected by aircraft during various field programs, experiments specifically designed for the validation of satellite remote sensing algorithms are needed to cover the range of surface and atmospheric conditions found in the polar regions.

\section{NARROW-BAND ALBEDO}

\subsection{Introduction}

Satellite-derived albedo values are useful for the estimation of the shortwave radiation balance, an important term in surface energy balance studies. For the study of spatial and temporal changes of surface albedo, satellites provide the only data source due to the inherent dynamic change in the sea ice cover. Over ice sheets, surface reflectance is a good indicator for classifying different surface types such as glacier ice and various snow facies. Snow and ice surfaces have relatively high albedo values $(50-90 \%)$, whereas the water surface has one of the lowest albedo values $(5-10 \%$ for high solar elevations). Snow and ice albedo depend on the solar incidence angle, snow grain size and geometry, impurities in the snow, water content in the snow cover, surface topography, and reflection angle. The albedo should be distinguished from the reflectivity, which refers to one specific wavelength.

\subsection{Algorithm}

The AVHRR multispectral narrow-band radiometric scanners with small fields of view need proper adjustments so that their data can be used to estimate top-of-theatmosphere albedo values (planetary albedo). The conversion of shortwave narrow-band data into average planetary albedo requires adjustment of the spectral expansion from filtered narrow-band to unfiltered broadband radiance of the shortwave spectrum from 0.24 to $4.2 \mu \mathrm{m}$. The planetary albedo for snow surfaces using AVHRR narrow-band satellite data can be derived through a multivariant regression analysis of Nimbus-7 earth radiation broad-band data and AVHRR narrowband data (Wydick and others, 1987). This statistical approach was done for NOAA-7, and still needs to be determined for the NOAA satellites 8 through 12 .

To derive surface albedo values from AVHRR narrow-band radiance measurements, the scattering and absorption of the atmosphere has to be included. This was successfully demonstrated by Koepke (1989) through numerical simulation that used a linear relationship between clear sky planetary albedo and surface albedo. The coefficients he derived are given as a function of solar zenith angle, optical depth of the atmosphere due to aerosols, integrated ozone content of the atmosphere for AVHRR channel 1 , and water vapor content for AVHRR channel 2. An alternate way to derive surface albedo values from AVHRR radiance measurements is to use a radiative transfer model such as LOWTRAN to correct for scattering and absorption as demonstrated by Haefliger and others (1993).

\subsection{Modeling and experimental measurements}

The knowledge of temporal changes in the spectral signal of snow is important for the understanding of albedo changes. The spectral albedo of snow in the solar spectrum is strongly a function of both the opticallyequivalent grain size, and concentration of absorbing impurities such as soot. Davis and others (1993) have shown, in their statistical analysis derived from stereological measurements, that the optically-equivalent grain size is a sphere with equal surface-to-volume ratio as the ice matrix. They used a one-dimensional energy and mass budget model that interacts with the snow cover and used outputs from this model as boundary conditions for a discrete ordinate radiative transfer model to predict the spectral bidirectional reflectance of the snowpack. These modeled results have still to be verified by in situ measurements.

Bidirectional reflectance of experimental measurements were compared with model results from a 48streams discrete-ordinate model (Steffen and Schweiger, unpublished). The model follows the Wiscombe and Warren (1980) approach where the two-stream approach is replaced with a discrete-ordinate solution (48 streams) to the radiative equation in order to allow modeling of the 
Bidirectional Distribution Reflectance Function (BDRF), The model assumes Mie-scattering of optically equivalent ice spheres. Small amounts of carbon soot had to be included in the medium to achieve a match between measured and modeled reflectances in the visible region. Comparison of model results with field measurements showed, in general, a good agreement if a soot amount of 0.30 parts per million weight (ppmw) was used. If the model run was performed with 0.00 ppmw soot amount, the spectral reflectance in the visible range $(0.3-0.8 \mu \mathrm{m})$ was approximately 0.1 higher than the in situ measurement.

Experimental measurements of optical properties were carried out in the laboratory for 70 snow samples collected in the Alps (Sergent and others, 1993). The carbon soot content was measured for all snow samples. The spectral reflectance between $0.4-1.0 \mu \mathrm{m}$ was obtained with a monochromator and comparison with the Wiscombe and Warren (1980) model showed good agreement. The study showed that crystal types and sizes (usually described in a subjective and simplified manner) have to be determined with great accuracy (i.e. statistical analyses of macro-photographs) to show a useful relation between snow grain-size, impurity content, and spectral reflectance.

Surface shortwave radiative fluxes over polar oceans can be obtained on a climatological basis from joint use of AVHRR and SSM/I data (Kergomard and others, 1993). The directional AVHRR radiances at the top of the atmosphere were simulated with a radiative transfer model with realistic atmospheric conditions, and compared in an iterative mode with average AVHRR visible radiances for cloudy pixels to derive optical thicknesses of the cloud cover. The SSM/I data provided the sea-ice concentrations and consequently, the surface reflectance. From the retrieved optical thickness, the incoming, absorbed and reflected solar fluxes $(0.2-2.8 \mu \mathrm{m})$ were computed by using a two-stream narrow-band model. The results agreed well with in situ measurements, but they are strongly dependent on cloud base temperature.

\subsection{Errors}

Prelaunch radiometric sensitivity of AVHRR visible and near infrared channels $(1,2)$ are determined with an integrating sphere by the manufacturer of the sensors to indicate their precision (Abel, 1990). The integrating sphere, however, has also a degrading sensitivity with time. Therefore, prelaunch calibrations, which are based on the intercomparison with the integrating sphere, will always have a relative error of up to a few per cent (i.e. the sphere calibration showed a drop in radiance of 10 $16 \%$ between 1983 to 1987 (Abel, 1990)).

A second and probably more important error source is the rate of degradation of the sensors over time. For the visible channel of NOAA 9, degradation rates of up to $6 \%$ per year were found (Staylor, 1990). Haefliger and others (1993) derived degradation rates of $0.6 \%$ per year for channel 1 and $4 \%$ per year for channel 2 for NOAA 1 based on a single comparison of in situ measured and modeled (AVHRR) reflectance values from the Greenland ice sheet.

In situ measurements of water vapor, aerosols, and ozone distribution with height are usually not available and must be parameterized. This parameterization bears another source of error which is difficult to estimate. Aerosol content can vary with time and space significantly in Arctic regions which can reduce the reflectance by up to $10 \%$ as reported by Lindsay and Rothrock (1993).

Satellite sensors with narrow field-of-view scanners measure the bidirectional reflectance at one or a few angles. If the reflecting surface, in our case snow, ice and water, is not Lambertian, than the measurements have to be corrected by the anisotropic reflectance factor (ARF), which is defined as the bidirectional reflectance normalized relative to the hemispheric reflectance. The first airborne bidirectional radiances of snow-covered surfaces have been reported by Hall and others (1993). For wet snow surfaces, the ARF at large solar zenith angles can be as large as 2 (Steffen, 1987). For new snow the ARF is close to 1 , and most likely can be neglected for AVHRR applications.

\subsection{Recommendations}

The overall accuracy of AVHRR-derived albedo values depends on a number of error sources, such as: pre-launch calibration, sensor degradation, uncertainty in aerosol, ozone and humidity concentrations over time and space, anisotropic reflectance of snow and ice, cloud contamination of pixels, and conversion from narrow-band albedo to broad-band albedo values. With the given uncertainties the expected error in AVHRR-retrieved surface albedo could be as large as $10 \%$. In a simple energy balance model this error $(\Delta$ albedo $=+10 \%)$ translates to a $3^{\circ} \mathrm{C}$ reduction in air temperature on an annual mean. This uncertainty is too large to be useful in climate sensitivity studies, and therefore, the following improvements are recommended.

1. AVHRR radiometric degradation has to be monitored carefully over time (no onboard calibration). Large scale reference areas of well known spectral reflectance properties, such as deserts or dry snow areas of ice sheets, could be used for frequent calibrations.

2. Conversion of planetary albedo to surface albedo is very sensitive to humidity, aerosol, and ozone concentrations in the atmosphere. In situ measurements of these variables are needed to characterize the atmospheric conditions to correct for absorption and scattering (two-stream models).

3. A statistical climatology of aerosol, ozone, and humidity distribution derived from satellite sounders is needed for different Arctic regions to reduce the uncertainty of large scale albedo retrieval.

4. Radiative transfer models with high spectral resolution should be used to correct for absorption and scattering based on statistical climatology as mentioned above (3).

\section{ICE CONCENTRATION}

\subsection{Introduction}

For some time AVHRR data have played an integral role in general ice-forecasting operations that produce maps of 
the ice edge and chart the location of large polynyas and wide leads (Hufford, 1981). Although effective automated means have not been developed to extract ice concentration from the AVHRR data stream, analysts who prepare operational ice charts through conventional manual interpretation methods use AVHRR images to map the ice edge on a routine basis.

More extensive use of AVHRR data to map ice concentration within the pack and to extract ice type information, whether through manual analysis, subjective classification techniques, or objective automated routines, has been limited, in large part by two factors. First, discrimination of thin ice from open water is next to impossible, particularly in the vicinity of open leads and polynyas where significant atmospheric water vapor and ice crystals bias radiances sensed from space. Second, interpretation of pixels that include mixtures of ice types that occur at subresolution scales, such as in leads or in areas where brash or small floes are common, is ambiguous.

\subsection{Algorithm and accuracy}

AVHRR ice edge locations for the Bering Sea in March 1988 were validated with Landsat imagery by Emery and others (1991a). The AVHRR ice edge location was found using a subjective threshold method to classify between water and ice. Results show the AVHRR edge to be consistently seaward of the Landsat edge. Mean deviation is $1.8 \mathrm{~km}$ in channel $2 \mathrm{data}$, and $2.9 \mathrm{~km}$ in channel 4 data with rms error of $3.2 \mathrm{~km}$ and $4.4 \mathrm{~km}$.

Derivation of ice concentration from AVHRR imagery requires that image pixels be classified as either water, ice, or cloud. Continuing progress in development of cloud masking algorithms as discussed in Section 2 will effectively reduce the problem to discrimination between ice and water in the near future. For clear cloud scenes, ice concentration can be achieved during winter months when good thermal contrast exists between ice and water surfaces.

Issues related to the derivation of ice concentration and ice type information from AVHRR imagery were addressed by two papers presented during this symposium. The first paper, by Key and others (1993), established a basis for evaluating the likelihood of observing a particular feature if its size with respect to the sensor beam spot, its contrast with adjacent ice, and if the conditions of the atmosphere are known. The authors base their analysis of contrast and atmospheric conditions on numerical simulations that use the LOWTRAN-7 model. Empirical approaches that degrade fine resolution Landsat images of leads to the coarser AVHRR resolution form the basis for analysis of scale relationships. Whereas work reported by Key and his co-authors was developed specifically to assess potential error in lead statistics derived from AVHRR imagery, the results have broader implications with respect to feature detection in general and with respect to derivation of ice type and ice concentration information.

The second paper, presented by Massom and Comiso (in press) demonstrated that frequency distributions formed by values of pixels in an AVHRR image are polymodal and carry information about the aerial extent of different ice types that are present in the scene. Massom and Comiso assign ice types to individual modes by interpreting relative brightnesses and temperatures that define pixels within the modes. The areal extent of each ice type in the scene then can be estimated as a function of the number of pixels within each mode. The method depends, of course, on reliable cloud-masking procedures to identify cloud pixels and remove them from consideration.

These two studies complement each other and, if future efforts are coordinated, could form the basis for deriving improved ice concentration estimates from AVHRR data. Massom and Comiso (in press), on one hand offer the potential for ice classification on a pixel-bypixel basis. The weakness of this approach is two-fold. First, pixels that include small features at subresolution scales (e.g., leads narrower than $1 \mathrm{~km}$ in the case of AVHRR nadir pixels) will be misclassified because their brightness represents the integrated radiance of more than one ice type - ice within the feature and ice adjacent to the feature. Second, variability of the atmosphere with time and location changes radiances measured from space and shifts the position of modes within the frequency distribution. Thresholds that define boundaries between ice types thus vary with respect to location within a single scene. Key and others (1993), on the other hand, provide the means to address these issues, to assess their impact on error inherent in AVHRR ice concentration products, and to adjust derived concentration estimates by appropriate values so that they reflect actual surface conditions more accurately. For example, frequency distributions used by the Massom-Comiso method can be modeled to determine the extent to which ice concentration retrievals are robust with respect to specific atmospheric profiles, varied assemblages of ice types, and collections of subresolution features (leads, floes, brash ice, and bodies of slush and shuga) that occur at different scales. Lead pixels, which can be identified using segmentation techniques and Hough transform methods (Duda and Hart, 1972; Fetterer and Holyer, 1989) and other pixels that are likely to include multiple surface types can be analyzed independently so as not to increase overall error. These mixed-surface pixels, which are most likely to include thin ice, can be analyzed further to narrow the range of possible surface types and feature widths required to produce the observed brightness signature.

\subsection{Recommendations}

Progress in three areas is requisite to utilize fully the results of the two studies mentioned above (Massom and Comiso, cited above; Key and others, 1993) and to improve ice concentration algorithms in general. Prerequisites for AVHRR-derived ice concentrations based on threshold methods are accurate ice surface and reflectance measurements (see Sections 3 and 4). Therefore, programs that provide for full characterization of radiometric properties of both sea ice and the atmosphere at AVHRR frequencies, improved methods of modeling the surface temperature field, and implementation of cloud-masking algorithms are needed. 
1. Ice properties. Optical and infrared signatures of different ice types and snow types must be described in terms of reflectance (possibly bidirectional reflectance), and thermal emissivity in order to define radiometric contrast for the work of Key and others, (1993), and to verify ice interpretations made in the course of the work of Comiso (unpublished). Limited data that describe some of these parameters have been published, both with regard to albedo of some types of sea ice (Grenfell and Perovich, 1984; Perovich, 1991) and bidirectional reflectance of snow (Dirmhirn and Eaton, 1975; Kuhn, 1985; Steffen, 1987). Additional measurements are needed to convert from broadband albedo to narrow-band albedo as measured by the AVHRR. Further, information is needed to characterize the transition from open water to thin ice, which is essential for monitoring heat and brine fluxes.

2. Characteristics of the atmosphere. Data that define atmospheric characteristics (water vapor, ice crystals, diamond dust, temperature) in polar regions, and thus the magnitude of bias these parameters contribute to space-based optical and infrared measurements of surface temperature and brightness, are not collected routinely except at widely scattered shore stations. Temporal and spatial variability is thought to be significant but is poorly documented at best and, in the central Arctic basin and in ice covered Antarctic waters, day-to-day variability is virtually unmonitored. Effort should be directed toward developing improved methods to model scattering, emission, and attenuation characteristics of the atmosphere using satellite data. Emphasis should be placed on innovative use of new sensors that will fly on EOS-era platforms. In particular, opportunities to employ space-based laser sensors as scatterometers to infer the presence of diamond dust should be explored.

3. Implementation of cloud detection algorithms. Automated ice concentration algorithms will require accurate cloud masking procedures. Progress toward effective cloud classification algorithms has been significant over the past five years. Efforts pursued by disparate groups of researchers should be coordinated to produce, within three years, a hybrid algorithm that embodies the best aspects of each approach, with transition to an operational system within five years.

\section{LEAD STATISTICS}

\subsection{Application}

Mapping leads in AVHRR imagery is a task with several applications. Heat flux through leads plays an inordinately large role in the Arctic heat budget. Lead width, lead density, the presence or absence of new ice in leads, and the orientation of leads relative to the wind are all important parameters when calculating surface fluxes. Ice rheology is revealed through large- and small-scale lead patterns. The opening and closing of leads is an effect of ice dynamics, which in turn affects the physical and biological oceanography of the upper ocean. Finally, the operational community would like to improve map and forecast lead width, orientation, and spacing, as these parameters influence the navigation of ships and submarines.

\subsection{Analysis}

The advantage of AVHRR for lead mapping is its synoptic view of Arctic basins and its frequency of coverage, which allows the study of lead formation in response to synoptic weather patterns and of regional and seasonal variations in lead distributions. Mapping leads is complicated by the same factors which challenge the use of AVHRR for ice concentration or surface temperature (see Sections 1-5). An added difficulty is that lead widths are generally assumed to follow a power law distribution. Relatively few leads are wide enough to fill the sensor field of view. The sensor resolution is about $1 \mathrm{~km}$ at nadir, and degrades to about $4 \mathrm{~km}$ at the scan limbs. Therefore virtually all "lead" pixels are mixtures of lead and surrounding ice, and the majority of leads are too narrow to be detected.

Walter and Overland (1993) illustrate that not all applications need wait for the full resolution of the issues above. Using AVHRR imagery from March and April 1991, they relate changes in lead patterns to wind fields. A long lead running diagonally across the Beaufort Sea revealed by sequential imagery acted as a slip boundary. North of this boundary, a diamond-shaped pattern of leads was present on two scales. At both scales, the angle of intersection between leads was about $30^{\circ}$. This pattern supports the theory of Erlingsson (1988), whose analysis of the mechanism of ice failure points to self-similarity of lead patterns. South of the slip boundary, a diamondshaped pattern is superimposed on a previously existing arch-shaped pattern. The dual pattern is related to changes in the wind field relative to the coastline and slip boundary to the north. This work emphasizes the feasibility of deducing ice mechanics from observable lead patterns, and the importance of the location relative to the direction of forcing of the slip, no-slip, or shear boundaries which confine lead pattern regimes.

Whether imagery is interpreted manually, as in the above studies, or automatically, some determination must be made of the conditions under which leads can be detected, with a view towards error analysis. In thermal data, the detectability of a lead is a function of the temperature contrast between a lead pixel and the surrounding ice, the size of the feature relative to the field of view of the sensor, and the effect of the intervening atmosphere. Key and others (1993) express detectability as a contrast ratio of the brightness temperature of leads measured at the sensor to that of thick ice. The ratio is a function of measurement scale (the ratio of the width of the lead to the sensor field of view) and of atmospheric optical depth. To model the function, the effect of measurement scale is obtained through degrading Landsat imagery of leads, while the effect of optical depth is obtained through modeling surface emissivities and radiative transfer using historical data. One result of the investigation into measurement scale is the observation 
that wide leads "grow" as imagery is degraded, while narrow leads disappear as averaging quickly reduces their contrast with surrounding ice. This implies that leadwidth statistics from AVHRR imagery should be adjusted for this effect. In investigating the effect of optical depth, the authors find that while brightness temperatures decrease slowly for four modeled ice thicknesses as aerosol optical depth increases, the brightness temperatures quickly converge with rising ice crystal optical depth. When ice crystal optical depth is greater than 3.0, it is difficult to distinguish open water in leads from ice.

Key and his coauthors (1993) supply a rational basis for selecting a threshold to segment leads in digital thermal imagery. An alternative to a straight threshold is the potential open water concept of Lindsay and Rothrock (1993). In this method, tie points for the reflectance or temperature of open water and of thick ice are used to establish the potential open water in each pixel. By applying a threshold to define lead pixels based on potential open water, no assumption need be made concerning the ice thickness or mixture of ice and open water in a pixel. The authors then derive lead widths using randomly placed transects and find that lead (and floe) widths are described by a negative exponential distribution over a range of regions and seasons. Lead width distributions obtained in this manner can serve as input to climate models.

Lindsay and Rothrock manually screened clouds to obtain data sets for studying regional distributions. This method is accurate but can be time consuming and can result in the loss of good data if the image interpreter is too conservative. However, constructing an algorithm for cloud detection in the Arctic is challenging (see Section 2). Rather than search for clouds directly, Eppler and Full (unpublished) developed a method of automatically distinguishing areas covered by leads from other surface types by applying edge filters to enhance the spatial signature of leads, and then classifying feature vectors drawn from cells in the filtered image. Lead-covered cells can then be supplied to a lead-statistics algorithm.

\subsection{Error analysis}

Errors in digital lead mapping are errors in detection (or how many lead pixels are missed), and errors in placement (or in the derived value of orientation, width, area, number, and spacing). Once lead pixels have been identified, errors in placement depend entirely upon the algorithm used to derive statistics. Key and others (1993) laid the groundwork for evaluating errors in detection when a thermal threshold was used to segment lead pixels by answering the question "What is the expected contrast between lead and ice for a given lead width and atmospheric condition?". If the variability of the temperature of background ice, the distribution of lead widths, and the variability of optical depth can be estimated, then the possibility of missed or falsely detected pixels can be evaluated. The change in field of view across the swath, and the effect of mapping from swath to gridded data, must also be taken into account.

\subsection{Recommendations}

To date, most lead mapping work has relied on manual analysis of imagery (e.g. Barry and others, 1989; Marko and Thomson, 1977) to yield information on ice rheology or lead formation. Using lead statistics as input to models has been largely unexplored, and is an application which puts more exacting requirements upon lead mapping techniques. Appropriate digital data sets and software tools can facilitate this work (see, for example, Lindsay and Rothrock, 1993), as will progress in the following areas:

1. Automated algorithms. The success of lead statistics as input to models will depend upon accurate climatologies. Automated analysis methods are needed to handle the volume of data required for good climatologies. Although not discussed at the Boulder symposium, two publications describe such algorithms. Key and others (1990b) use a rulebased system to decide which features constitute leads and to produce distributions of width, length and orientation. One advantage is that rules for lead definition can be fitted to the application. Fetterer and others (1990) extract distributions of lead width, orientation, and spacing using a Hough transform-based line finding technique. Rather than depending solely on reflectance or thermal contrast to identify lead pixels, algorithms can use the linearity of leads. This is an important advantage given the variability in lead contrast. Of course, errors in derived lead parameters due to the algorithm itself must be accounted for. This can be an onerous task when the algorithm involves many processing steps, and was not performed in the cited work.

2. Lead width distributions. Two opportunities for extending AVHRR measurements to smaller scales were put forth by symposium participants. The scale-invariant nature of lead patterns observed by Walter and Overland (1993) suggests that if the boundaries of pattern regimes are identified, smaller scale patterns can perhaps be inferred. Key and others (1993) attempted to use lead width distribution from AVHRR to predict the true (full-scale) width distribution. Results were overly sensitive to the AVHRR data used as input, but accuracy could be improved using coincident SAR or Landsat data to fix the predicted distribution at the high end. More comparisons of AVHRR with higher resolution data are called for in order to understand better the relationship between the pattern of large and small scale leads and the variability in lead width distributions.

3. Access to more data. One year (1989) of a five-year data set of digital AVHRR data covering the entire Arctic as frequently as every three days has been assembled under the sponsorship of the Office of Naval Research (Fetterer and Hawkins, 1993). The data set was compiled to address the problem many researchers have in finding sufficient quantities of processed, cloud-free data over a wide range of seasons and regions. Broader use of this data set for lead studies and more detailed correlation analysis with Landsat, Spot and other high resolution images are encouraged. 


\section{SEA-ICE MOTION}

\subsection{Applications}

Ice motion measured from sequential satellite imagery is applicable to a range of geoscientific problems. In the momentum balance for sea ice, the water stress term depends directly on ice velocity, and the divergence of internal ice stress depends on the spatial gradient of ice velocity. The ice mass balance equation has an advective term, given by ice velocity. New ice production and the resulting brine flux to the ocean surface depends on spatial differences of ice velocity or, for shore leads and polynyas, on the offshore component of ice velocity. The piling of ice mass into ridges depends on convergent and shearing ice motions. The ice velocity field is a strong boundary condition (certainly more directly observable than stress) on the ocean surface. Ice velocity data enhance operational forecasts of polynya and lead openings or closings, and of the advection of multiyear floes into navigation lanes. The motivation for acquiring ice motion data is high.

\subsection{Algorithms}

Ice tracking consists first of identifying the same ice feature in each of two sequential images, A and B, and second, of specifying the geographic location of these points in the two images. Each of these two steps has an algorithm. The algorithm most widely used for tracking ice in AVHRR imagery is maximum cross-correlation. This technique finds a local maximum in the crosscorrelation as a function of the spatial lag (in $\mathrm{x}$ and $\mathrm{y}$ ) between the two scenes (Ninnis and others, 1986; Emery and others, 1991b; Zhang, 1991). The spatial lag of the peak correlation indicates the distance the ice has moved. Edge feature tracking and floe boundary tracking algorithms applied recently to SAR ice tracking (McConnell and others, 1991; Banfield, 1991) have generally not been applied to AVHRR.

Geolocation is accomplished by a second algorithm. The procedure is to adjust parameters in an orbit model to give correct coordinates of known ground points, and to use the new orbit information to compute new locations for all points in the image (Baldwin and Emery, 1993; Emery and others, 1989). When comparing two images as when tracking ice, one image is sometimes "nudged" (translated uniformly) so that fixed (land) portions of the image more perfectly overlay each other. The procedure of warping one image to correspond to another, which is common for overlaying, say, Landsat scenes to maps, is inappropriate for AVHRR sea-ice tracking; in sea-ice scenes with land in a portion of the scene, warping can cause severe distortions away from land.

Three papers presented at the symposium dealt with ice motion or image geolocation. Heacock and others (1993) discuss the difficulties of tracking seasonal ice off the coast of Newfoundland and Labrador. In addition to the usual problem of cloud cover, floe sizes well below the resolution of AVHRR cause difficulty to both correlation and feature tracking algorithms; the tracker must recognize the patchiness in the ice cover. Consequently, tracking works best for quite short time intervals, 6 to 48 hours. Feature identification errors average $2.5 \mathrm{~km}$. Using a "nudging" navigation technique, they obtain geolocation errors of about $2.7 \mathrm{~km}$.

Emery and Fowler (1992) reviewed an operational version of a correlation ice tracker developed for the Navy/NOAA Joint Ice Center (Emery and others, 1991b). The algorithm adds a spatial filtering technique, eliminates Fast Fourier Transforms to compute correlations, and allows for irregular correlation areas near coastlines. Ice velocities computed from different AVHRR channels (2 and 4) show almost no difference. The use of low-resolution (simulated GAC) imagery provides roughly one quarter of the useable motion vectors found with the higher resolution LAC images, so the field of motion loses much spatial detail. Clearly, Fram Strait and the Greenland Sea are ideal for AVHRR ice motion observations: the ice motions are large enough to swamp the errors, and there is land on both sides of the strait to maximize the geolocation accuracy.

Baldwin and Emery (1993) describe a geolocation algorithm that computes from two or more ground control points both the satellite orientation (roll, pitch, and yaw) and the satellite position at a known time (the time the image is acquired). The algorithm can compute both the earth locations of image pixels ("satellite perspective" or orbital data) and resampled pixels on a chosen geographic projection ("indirect navigation" or geolocated imagery). The geolocation accuracy of the method is $1 \mathrm{~km}$. The paper describes the steps required in the calculation, and techniques used to make the computing more efficient.

\subsection{AVHRR compared with other motion data sources}

Ice motion data are also available from drifting buoys and from other satellites including those with synthetic aperture radar (SAR). In light of SAR's substantially higher resolution and lower geolocation errors, one can ask what role AVHRR should play in a routine ice tracking capability. At present AVHRR coverage has some advantages to that of SAR. AVHRR has a wide swath (about $2750 \mathrm{~km}$ ); it provides an occasionally excellent basin-scale perspective. AVHRR HRPT is received at many ground stations giving a great deal of Arctic data, but only some 10 to $30 \%$ is cloud-free. The ERS-1 and JERS-1 SAR satellites provide narrow swath coverage $(100 \mathrm{~km})$ leaving many areas unseen. Their pixel spacing is far superior to AVHRR's $1 \mathrm{~km}$ or greater $-12.5 \mathrm{~m}$ at high resolution and $100 \mathrm{~m}$ at low resolution. The Optical Line Scanner on the DMSP satellites with a $600 \mathrm{~m}$ pixel spacing is another excellent tool for testing the accuracy of ice motion data. The advangtage of buoys is the length and continuity of their data record; their positional accuracy (equivalent to $\sigma_{\mathrm{g}}$ ) is several hundred meters, sufficient to provide valuable comparisons with AVHRR ice motions.

\subsection{Errors}

Errors in the ice motion are described as uncertainty in the geographic location of any pixel in either scene; and 
uncertainty in identification of the same feature in both scenes. Although error models are generally lacking in the literature, both the geolocation error and the feature identification error have apparently been taken to be zero-mean, Gaussian errors with standard deviations $\sigma_{\mathrm{g}}$ (for geolocation) and $\sigma_{\mathrm{f}}$ (for feature identification). We take discussion of "errors" in the literature to refer to these two standard deviations. The geolocation error applies to the feature position in each image; the feature identification error applies to the feature position in image $B$, the feature being defined by its position in image A. Although many geophysical applications require velocities, the actual measurement is of position and its temporal and spatial differences. A component of displacement between images $\mathrm{A}$ and $\mathrm{B}$ is

$$
u=\left(x_{\mathrm{B}}-x_{\mathrm{A}}\right)
$$

and has an error with a standard deviation, assuming uncorrelated geolocation errors, of

$$
\sigma u=\left(2 \sigma_{\mathrm{g}}^{2}+\sigma_{\mathrm{f}}^{2}\right)^{\frac{1}{2}}
$$

The relative motion between two features labeled 1 and 2 is used to estimate velocity gradients and strain; we refer to it generically as stretching. It has the the form

$$
\Delta u=u_{2}-u_{1}
$$

and has an error with standard deviation

$$
\sigma \Delta u=\left(4 \sigma_{\mathrm{g}}^{2}+2 \sigma_{\mathrm{f}}^{2}\right)^{\frac{1}{2}}
$$

If the points are close together, the geolocation errors for points 1 and 2 tend to cancel, and this error is reduced towards

$$
\left(2 \sigma_{\mathrm{f}}^{2}\right)^{\frac{1}{2}}
$$

The values quoted for these errors have been: $\sigma_{\mathrm{g}}$ from 1 to $5 \mathrm{~km}$, and $\sigma_{\mathrm{f}}$ from 0.9 to $2.5 \mathrm{~km}$; see Table 2 . AVHRR ice tracking has been performed almost exclusively with HRPT and LAC data with a resolution varying from $1.1 \mathrm{~km}$ at nadir to $4 \mathrm{~km}$ at the swath edge. These values give a displacement error $\sigma_{\mathrm{u}}$ (from Equation 2) between 1.8 and $7.5 \mathrm{~km}$, an upper bound for the stretching error $\sigma \Delta u$ (from Equation 4) between 2.5 and $10.6 \mathrm{~km}$, and a

Table 2. Values for geolocation and feature identification errors, in $\mathrm{km}$

Reference

$\sigma_{\mathrm{g}} \quad$ Reference

$\sigma_{\mathrm{f}}$

Clark and LaViolette 2 to 4

Emery and others

(1989)

Bordes and others

(1992)

Heacock and others

(1993) lower bound for the stretching error $\sigma \Delta u$ (from Equation 5) between 1.3 and $3.5 \mathrm{~km}$. These errors are quite significant compared to typical daily ice motions between several $\mathrm{km}$ and 10 or $20 \mathrm{~km}$, and stretching of roughly 1 to $10 \mathrm{~km}$ per $100 \mathrm{~km}$ of spatial separation. Many of the low estimates of error magnitudes are for ideal cases: ice near land and near swath centers with a nadir view and the smallest image pixels.

For AVHRR ice tracking in particular, it seems possible to make a clear statement about desired error size. If the geolocation and feature identification errors can each be limited to about $1 \mathrm{~km}$, the motion data are quite useful scientifically. Measurements of this accuracy produce ice displacements with errors of $1.7 \mathrm{~km}$ and stretching errors (over a short baseline, from Equation 5) of $1.4 \mathrm{~km}$.

\subsection{Recommendations}

Because errors can render ice motion measurements scientifically useless, effort should be directed at documenting and testing errors more thoroughly, and in reducing them to their lower limits. It would be good practice to attach an error estimate to every ice motion vector and to treat this error as a function both of crosstrack position within the swath, and of distance from ground points used to geolocate the image.

1. The feature identification error $\sigma_{\mathrm{f}}$ increases with scan angle. Near the swath edges cross-track pixel size is $4 \mathrm{~km}$. The practice has been to use data within a vaguely defined "center of the swath", and ignore this variation. We recommend that feature identification error be treated as a function of crosstrack position in LAC and HRPT data, and that this dependency be documented.

2. Geolocation error $\sigma_{\mathrm{g}}$ is believed to increase with distance from ground points. Some test of this notion is needed. Tests could be run with continental scenes, treating them as sea-ice images with land in only a portion of the scene. If errors are clearly shown to grow with distance from land, this behavior should be incorporated in error estimates for all ice motion vectors.

3. The errors in ice motion data are generally errors in position, not errors in the time of the images. Dividing displacements by the time interval between images to compute velocity can obfuscate the discussion of errors. We recommend using position and displacement, not velocity, when discussing errors.

4. Validation of the combination of geolocation and feature identification error should be accomplished by comparison with buoy drift data and with SAR satellite ice tracking.

\section{ICE SHEET STUDIES}

\subsection{Introduction}

AVHRR data can be used for ice sheet studies for three main purposes: (1) surface temperature, discussed in 
Section 3; (2) surface morphology of the ice sheet; (3) calving of the ice fronts. Recently work has been done on deriving accumulation rate and snow grain-size from AVHRR data, but error estimations are needed before applying these methods over large areas of the ice sheet.

\subsection{Surface morphology}

Morphological studies include the identification of surface features (Casassa and others, 1991) and the characterization of different modes of ice flow (Bindschadler and Vornberger, 1990; Seko and others, 1993). This information can indicate present and past flow conditions of the ice and is especially important for improving the map coverage of large ice masses.

Since surface features do not change rapidly with time, frequent coverage is not required, but accurate georeferencing is essential for determining the location. The fine spatial scale of these features, however, requires AVHRR of the highest spatial resolution (LAC near the swath center) and highest radiometric resolution (10-bit). With these resolutions, topographic roughness of approximately $10^{-3}$ over horizontal scales of more than a few kilometers can be detected. Topographic features are revealed most effectively at orthogonal sun illumination. Thus, the need for imagery of the same area at various sun azimuths is recommended. The permanence of these surface features affords the opportunity for mosaicking different images to obtain the best surface information. Visible and near-infrared bands are best for this purpose.

\subsection{Calving Rate}

Monitoring ice fronts with AVHRR data on a periodic basis permits the detection of large iceberg calving events (Ferrigno and Gould, 1987; Keys and others, 1990). AVHRR data is also useful for monitoring the position of the ice front and comparing with historical data for deriving calving rates with accuracy of $0.2 \mathrm{~km} \mathrm{a}^{-1}$. With this kind of accuracy it is possible to monitor fast-moving ice fronts with AVHRR data over periods of 5-10 years to obtain reliable calving rate information.

High spatial resolution is needed and accurate georeferencing is essential. Visible and near-infrared channels are preferred, although ice fronts also appear clearly in thermal infrared bands, which permits monitoring during winter at high latitudes.

\subsection{Potential for other ice sheet studies}

In the interior of East Antarctica AVHRR brightness patterns correlates strongly with surface topography (Seko and others, 1993). Since accumulation rate is closely related to topography, AVHRR data can be used to extend the sparse data on accumulation rate from East Antarctica. However, an assessment of the accuracy of this method is needed. The multispectral dependence of reflectivity on snow grain-size has permitted the quantification of grain-size from Landsat-TM data (Bordelles and Fily, 1993). This method could be used with AVHRR visible and near-infrared bands for deriving snow grain-size spatially. This can provide an independent measure of surface emissivity and help in the extraction of other parameters (accumulation rate, physical temperature) from passive microwave data. Error estimation is needed for this method as well.

\subsection{Recommendations}

1. AVHRR of the highest spatial resolution (LAC near the swath center) and highest radiometric resolution (10-bit) are required to resolve surface features.

2. Accurate georeferencing is essential for determining the location as ice sheets have only a few fixed reference points.

3. Imagery of the same area at various sun azimuths and orthogonal sun illumination are needed to reveal topographic features most effectively.

\section{CONCLUSIONS}

The previous seven sections discussed the great potential of AVHRR applications, but also showed major shortcomings which need to be taken into account for polar applications. Today, a major emphasis in polar research is directed towards monitoring surface parameters that describe present climate conditions and help us understand how the climate system functions. AVHRR data have the potential to provide a rich data stream bearing on polar conditions and processes, but these data have been underutilized, due mainly to the inaccessibility of calibrated, geolocated LAC and HRPT data sets. Many applications, such as cloud masking, surface temperature, lead statistics, ice concentration, and ice motion, require multi-temporal data sets for process studies and for reducing the uncertainty in derived surface parameters. Initial steps to provide such data sets have been made by the ONR-supported AVHRR 1989 data set (Fetterer and Hawkins, 1993).

Retrieval of ice surface temperature shows promise and would represent a significant contribution to the field, considering the current lack of spatially detailed measurements in the polar regions. This might be the most important application of AVHRR (and similar) data in the polar regions, since some of the other applications can be more effectively met by other satellite systems. But retrieved data have not been yet validated, although the estimated accuracy is believed to be between within 1 and $4 \mathrm{~K}$. Atmospheric models (radiative transfer) to account for absorption and emission of thermal radiation are in place, but additional information on ice crystal precipitation and aerosol distribution are needed to reduce the uncertainty in IST retrieval. The same parameters are needed for an improved albedo retrieval. Currently, narrow-band albedo can be retrieved for cloud free pixels within an accuracy of $1-4 \%$ for summer conditions (no ice crystal precipitation) if coincident atmospheric profile measurements are available. Ice concentration algorithms make use of surface reflectance and surface temperature information and consequently also need improved atmospheric input data to characterize temporal and spatial variability of water vapor, diamond dust, and ice crystals in conjunction with improved cloud masking algorithms. Emphasis should 
be placed on use of a combined sensor algorithm (i.e. passive microwave, thermal and visible, synthetic aperture radar) to circumvent present problems with cloud masking in the visible and thermal spectra.

Lead detection has been highly successful for some individual case studies, but estimates of lead statistics and other parameters required as input to models have to be refined. Additional research is needed to understand the pattern of large-scale and small-scale leads and the variability in lead-width distribution (i.e., AVHRR in combination with Landsat or SAR data). Ice motion retrieval depends in the first order on the geolocation accuracy of the AVHRR data. This accuracy was reported to be between $0.8-4 \mathrm{~km}$, depending on location and the presence of distinct geographical features. Feature identification is also crucial for ice tracking, and presently this accuracy is quoted to be between 0.9 $2.5 \mathrm{~km}$, also depending on the authors. Assuming a geolocation and feature identification error of $1 \mathrm{~km}$, and ice displacement error of $1.7 \mathrm{~km}$, an ice stretching error of $1.4 \mathrm{~km}$ could be expected.

Having taken stock of AVHRR polar algorithms, we see three major objectives for advancing the scientific utility of AVHRR data. First, development of unsupervised cloud masking techniques and subsequent evaluations of their accuracy must be attacked with great energy. Cloud masking is essential for all retrievals of surface parameters, but today there is no operational polar cloud masking algorithm in place with sufficient accuracy $(>90 \%)$ to be useful to the research and operations communities. Night-time cloud masking is far from being operational, and it is too early even to estimate the accuracy that may be achievable. In a real sense, the development of all-surface parameter algorithms for large scale studies is held up by the lack of adequate cloud masking.

* We recommend that the different polar cloud masking groups work closely together to guarantee coordination and timely development of satisfactory algorithms.

Second, clear statements of the accuracies of parameter retrievals must be provided by algorithm developers so that the scientific community can assess in the geophysical data derived from the sensor. Algorithms accepted by a knowledgeable portion of the community must be widely disseminated. The present situation is that the validity of some retrievals is open to question; the scientific community will not support devoting resources to the processing of data in which they have no basis for confidence.

* We recommend that funding agencies devote more resources to validation work and to careful error estimation as part of research on these algorithms.

Third, acquisition and processing of polar AVHRR data must become a coordinated effort between the science community, receiving stations and data centers, and the funding agencies to guarantee a long-term commitment to the collection and distribution of high quality data. The various receiving stations and their associated archives should begin a dialogue among themselves and with their scientific users and funding agencies to establish uniform data standards and even common formats for geophysical data products. Algorithms to be used must be chosen by or with the consent of the scientific community. Such action will free geoscientists from the need to postprocess satellite data, and allow them to concentrated on the study of geophysics. If such standardization among stations and archives is not possible, then a single site for archiving polar high-resolution AVHRR data should be identified and funded to collect data from various stations and to convert it to geophysical products of common formats and known accuracies.

* We recommend a workshop be convened to provide a forum for representatives of all polar receiving stations and representatives of the scientific user community to investigate ways to implement a common data catalog, and to standardize AVHRR data products.

* We recommend that a data center be identified to coordinate acquisition, processing, and distribution between all receiving stations. It would be desirable to have the current data acquisitions accompanied by suitable ancillary data sets that could be used to improve interpretation of the data and to validate derived products.

* We recommend careful evaluation of information content of AVHRR LAC versus AVHRR GAC data. GAC data is more suitable for global analysis in the context of monitoring and climate change studies.

Until such steps are taken, this valuable data source will continue in its present state of underutilization.

\section{REFERENCES}

Abel, P. 1990. Prelaunch calibration of NOAA-11 AVHRR visible and near IR channels. Remote Sensing Environ., 31, 227-229.

Baldwin, D. G. and W.J. Emery. 1993. A systemized approach to AVHRR image navigation. Ann. Glaciol., 17 (see paper in this volume).

Banfield, J. 1991. Automated tracking of ice floes: a stochastic approach. IEEE Trans. Geosci. Remote Sensing, 29(6), 905-911.

Barry, R. G., M. W. Miles, R. C. Cianflone, G. Scharfen and R. C. Schnell. 1989. Characteristics of Arctic sea ice from remote-sensing data and their relationship to atmospheric processes. Ann. Glaciol., 12, 9-15.

Bindschadler, R.A. and P. L. Vornberger. 1990. AVHRR imagery reveals Antarctic ice dynamics. Eos, 71(23), 741-742.

Bordes, P., P. Brunel and A. Marsouin. 1992. Automatic adjustment of AVHRR navigation. 7. Atmos. Oceanic Technol., 9, 15-27.

Bourdelles, B. and M. Fily. 1993. Snow grain-size determination from Landsat imagery over Terre Adélie, Antarctica. Ann. Glaciol., 17 (see paper in this volume).

Casassa, G. and J. Turner. 1991. Dynamics of the Ross 
Ice Shelf. Eos, 72(44), 473-481.

Clark, J.R. and P.E. LaViolette. 1981. Detecting the movement of oceanic fronts using registered Tiros- $\mathrm{N}$ imagery. Geophys. Res. Lett., 8(3), 229-232.

Davis, R. E., A. W. Nolin, R. Jordan and J. Dozier. 1993. Towards predicting temporal changes of the spectral signature of snow in visible and near-infrared wavelengths. Ann. Glaciol., 17 (see paper in this volume).

Dirmhirn, I. and F. D. Eaton. 1975. Some characteristics of the albedo of snow. F. Appl. Meteorol., 14, 375-379.

Duda, R. O. and P.E. Hart. 1972. Use of the Hough transform to detect lines and curves in pictures. Commun. ACM, 15(1), 11-15.

Ebert, E. 1987. A pattern recognition technique for distinguishing surface and cloud types in the polar regions. F. Climate Appl. Meteorol., 26(10), 1412-1427.

Emery, W.J., J. Brown and Z. P. Nowak. 1989. AVHRR image navigation: summary and review. Photogramm. Eng. and Remote Sensing, 55(8), 1175-1183.

Emery, W.J., M. Radebaugh, C. W. Fowler, D. Cavalieri and K. Steffen. 1991a. A comparison of sea ice parameters computed from Advanced Very High Resolution Radiometer and Landsat satellite imagery and from airborne passive microwave radiometry. $\mathcal{J}$. Geophys. Res., 96(C12), 22,075-22,085.

Emery, W.J., C.W. Fowler, J. Hawkins and R.H. Preller. 1991b. Fram Strait satellite image-derived ice motions. 7. Geophys. Res., 96(C3), 4751-4768. (Correction: J. Geophys. Res., 96(C5), 8917-8920, 1991.)

Erlingsson, B. 1988. Two-dimensional deformation patterns in sea ice. J. Glaciol., 34(118), 301-308.

Ferrigno, J.G. and W.G. Gould. 1987. Substantial changes in the coastline of Antarctica revealed by satellite imagery. Polar Rec., 23(146), 577-583.

Fetterer, F. and J. Hawkins. 1993. Data set of Arctic AVHRR imagery for the study of leads. Ann. Glaciol., 17 (see paper in this volume).

Fetterer, F. M. and R.J. Holyer. 1989. A Hough transform technique for extracting lead features from sea ice imagery. IGARSS'89. 12th Canadian Symposium on Remote Sensing. Quantitative remote sensing, an economic tool for the nineties, Vancouver, Canada, July 10-14, 1989. Volume 2, 1125-1128.

Fetterer, F., A. E. Pressman and R. L. Crout. 1990. Sea ice lead statistics from satellite imagery of the Lincoln Sea during the ICE SHELF Acoustic Exercise, Spring 1990. Stennis Space Center, MS. (NOARL Technical Note 50.)

Gallegos, S. C., J. D. Hawkins and C. F. Cheng. 1993. Cloud screening in AVHRR digital data over Arctic regions. Ann. Glaciol., 17 (see paper in this volume).

Grenfell, T.C. and G.A. Maykut. 1977. The optical properties of ice and snow in the Arctic Basin. $\mathcal{J}$. Glaciol., 18(80), 445-463.

Grenfell, T.C. and D. K. Perovich. 1984. Spectral albedos of sea ice and incident solar irradiance in the southern Beaufort Sea. 7. Geophys. Res., 89(C3), 35733580 .

Haefliger, M., K. Steffen and C. Fowler. 1993. AVHRR surface temperature and narrow-band albedo comparison with ground measurements for the Greenland ice sheet. Ann. Glaciol., 17 (see paper in this volume).

Hall, D. K., J. L. Foster, J. R. Irons and P. W. Dabney.
1993. Airborne bidirectional radiances of snow surfaces in Montana, U.S.A. Ann. Glaciol., 17 (see paper in this volume).

Heacock, T., T. Hirose, F. Lee, M. Manore and B. Ramsay. 1993. Sea-ice tracking on the east coast of Canada using NOAA AVHRR imagery. Ann. Glaciol., 17 (see paper in this volume).

Hufford, G. L. 1981. Sea ice detection using enhanced infrared satellite data. Mariners Weather Log, 25(1), 1-6.

Kergomard, C., B. Bonnel and Y. Fouquart. 1993. Retrieval of surface radiative fluxes on the marginal zone of sea ice from operational satellite data. Ann. Glaciol., 17 (see paper in this volume).

Key, J. 1990. Cloud cover analysis with Arctic Advanced Very High Resolution Radiometer data. 2. Classification with spectral and textural measures. 7. Geophys. Res., 95(D6), 7661-7675.

Key, J. and M. Haefliger. 1992. Arctic ice surface temperature retrieval from AVHRR thermal channels. J. Geophys. Res., 97(D5), 5885-5893.

Key, J., A.J. Schweiger and J.A. Maslanik. 1990. Mapping sea ice leads with a coupled numeric/ symbolic system. ACSM/ASPRS Proceedings, Denver, Colorado, March 18, 1990. Vol.4, 228-237.

Key, J., R. Stone, J. Maslanik and E. Ellefsen. 1993. The detectability of sea-ice leads in satellite data as a function of atmospheric conditions and measurement scale. Ann. Glaciol., 17 (see paper in this volume).

Keys, H.J.R., S.S. Jacobs and D. Barnett. 1990. The calving and drift of iceberg B-9 in the Ross Sea, Antarctica. Antarct. Sci., 2(3), 243-257.

Kidwell, K. B. 1991. NOAA polar orbiter data users guide. Washington, DC, National Oceanic and Atmospheric Administration.

Koepke, P. 1989. Removal of atmospheric effects from AVHRR albedos. 7. Appl. Meteorol., 28, 1341-1348.

Kuhn, M. 1985. Bidirectional reflectance of polar and alpine snow surfaces. Ann. Glaciol., 6, 164-167.

Lindsay, R. R. and D. Rothrock. 1993. The calculation of surface temperature and albedo of Arctic sea ice from AVHRR. Ann. Glaciol., 17 (see paper in this volume).

McClain, E. P., W. G. Pichel and C. C. Walton. 1985. Comparative performance of AVHRR-based multichannel sea surface temperatures. 7. Geophys. Res., 90, 11,587-11,601.

McConnell, R., R. Kwok, J. C. Curlander, W. Kober and S. S. Pang. 1991. Y-s correlation and dynamic time warping; two methods for tracking ice floes in SAR images. IEEE Trans. Geosci. Remote Sensing, 29(6), 1004 1012.

Marko, J. R. and R. E. Thomson. 1977. Rectilinear leads and internal motions in the ice pack of the western Arctic Ocean. 7. Geophys. Res., 82(6), 979-987.

Maslanik, J. and J. Key. 1993. Comparison and integration of ice-pack temperatures derived from AVHRR and passive microwave imagery. Ann. Glaciol., 17 (see paper in this volume).

Massom, R.A. and J. C. Comiso. In press. The characterization of new sea ice formation and the determination of surface temperature using Advanced Very High Resolution Radiometer data. 7. Geophys. Res.

Minnis, P. and E. F. Harrison. 1984. Diurnal variability 
of regional cloud and clear-sky radiative parameters derived from GOES data. I. Analysis method. $\mathcal{F}$. Climate Appl. Meteorol., 23, 993-1011.

Ninnis, R. M., W.J. Emery and M.J. Collins. 1986. Automated extraction of pack ice motion from advanced very high resolution radiometer imagery. 7. Geophys. Res., 91 (C9), 10,725-10,734.

Perovich, D. K. 1991. Seasonal changes in sea ice optical properties during fall freeze-up. Cold Reg. Sci. Technol., 19(3), 261-273.

Rees, W. G. and I-I. Lin. 1993. Texture-based classification of cloud and ice-cap surface features. Ann. Glaciol., 17 (see paper in this volume).

Rossow, W. B. and R.A. Schiffer. 1991. ISCCP cloud data products. Bull. Am. Meteorol. Soc., 72, 2-20.

Rossow, W.B., L.C. Gardner and A.A. Lacis. 1989. Global, seasonal cloud variations from satellite radiance measurements. Part I. Sensitivity of analysis. 7. Climate, 2, 419-458.

Saunders, R. W. and K. T. Kriebel. 1988. An improved method for detecting clear sky and cloudy radiances from AVHRR data. Int. F. Remote Sensing, 9, 123-150.

Seko, K., T. Furukawa, F. Nishio and O. Watanabe. 1993. Undulating topography on the Antarctic ice sheet revealed by NOAA AVHRR images. Ann. Glaciol., 17 (see paper in this volume).

Sergent, C., E. Pougatch, M. Sudul and B. Bourdelles. 1993. Experimental investigation of optical snow properties. Ann. Glaciol., 17 (see paper in this volume).

Serreze, M.C., J.A. Maslanik, G.R. Scharfen, R.G. Barry and D. A. Robinson. 1993. Interannual variations in snow melt over Arctic sea ice and relationships to atmospheric forcings. Ann. Glaciol., 17 (see paper in this volume).
Staylor, W.F. 1990. Degradation rates of the AVHRR visible channel for the NOAA 6,7 , and 9 spacecraft. $\mathcal{F}$. Atmos. Oceanic Technol., 7, 411-423.

Steffen, K. 1987. Bidirectional reflectance of snow at 500$600 \mathrm{~nm}$. International Association of Hydrological Sciences Publication 166 (Symposium at Vancouver 1987 Large Scale Effects of Seasonal Snow Cover), 415-425.

Stowe, L. L., H. Y. M. Yeh, T. F. Eck, C. G. Wellemeyer and H.L. Kyle. 1989. Nimbus-7 global cloud climatology. Part I. First-year results. F. Climate, 2, 671-709.

Walter, B.A. and J.E. Overland. 1993. The response of lead patterns in the Beaufort Sea to storm-scale wind forcing. Ann. Glaciol., 17 (see paper in this volume).

Welch, R. M., K. Kuo and S. K. Sengupta. 1990. Cloud and surface textural features in polar regions. IEEE Trans. Geosci. Remote Sensing, 28(4), 520-528.

Welch, R. M., S.K. Sengupta, A. K. Goroch, P. Rabindra, N. Rangaraj and M.S. Navar. 1992. Polar cloud and surface classification using AVHRR imagery: an intercomparison of methods. F. Appl. Meteorol., 31, 405-420.

Wiscombe, W.J. and S. G. Warren. 1980. A model for the spectral albedo of snow. I. Pure snow. F. Atmos. Sci., 37(12), 2712-2733.

Wydick, J.E., P.A. Davis and A. Gruber. 1987. Estimation of broadband planetary albedo from operational narrowband satellite measurements. NOAA Tech. Rep. NESDIS 27.

Zhang, H. 1991. Parameter retrieval algorithms and data analysis system for sea ice remote sensing. (Ph.D. thesis, Technical University of Denmark.)

The accuracy of references in the text and in this list is the responsibility of the authors, to whom queries should be addressed. 